\title{
The Super B factory projects
}

\section{Christoph Schwanda*}

Institute of High Energy Physics, Austrian Academy of Sciences

E-mail: schwanda@hephy. oeaw.ac.at

As the era of the $B$ factories Belle and BaBar is coming to an end, two upgrade projects, SuperKEKB/Belle-II in Japan and SuperB in Italy, have been proposed to probe the theory of Cabibbo, Kobayashi and Maskawa to an even higher degree of precision and to search for New Physics at the loop level. In this article, we briefly review the physics case of the Super $B$ projects and discuss the status of the accelerator and detector design.

12th International Conference on B-Physics at Hadron Machines - BEAUTY 2009 September 07 - 112009

Heidelberg, Germany

\footnotetext{
* Speaker.
} 


\section{Introduction}

The era of the $B$ factory experiments Belle [1] and BaBar [2] is now coming to an end: by December 2009, BaBar at PEP-II (stopped in 2008) has accumulated a data set of $557 \mathrm{fb}^{-1}$ and Belle at KEKB has reached an integrated luminosity of $1018 \mathrm{fb}^{-1}$. These data sets, mostly taken at the $\Upsilon(4 S)$ resonance, led to many significant results, some of them expected (discovery of $C P$ violation in $B$ meson decays, measurement of the sides and angles of the unitarity triangle, discovery of $D$ mixing), some of them not (observation of new charmonium and charmonium-like states, hints for New Physics). Most importantly, the $B$ factories have confirmed the theory of $C P$ violation of Makoto Kobayashi and Toshihide Maskawa [3], which led to their physics Nobel Prize in 2008.

This success has motivated the Super $B$ factory projects SuperKEKB/Belle-II in Japan and SuperB in Italy that will probe the CKM theory with almost two orders of magnitude more data. Precision measurements of the CKM unitarity triangle will restrict the parameter space for Physics beyond the Standard Model and guide direct searches at high energies, or even provide evidence for New Physics. In this articles, we will briefly review the physics case of the Super $B$ factories and then present the current status of both projects regarding the accelerator and detector design.

\section{Physics Case}

As the LHC experiments are starting to investigate New Physics in the TeV range, it is crucial that these direct searches are guided and complemented by precision measurements that can look for the virtual production of new particles in quantum loops. Flavor physics is a rich laboratory for this kind of searches as flavor changing neutral current processes such as $B \rightarrow X_{s} \gamma$, neutral mesonantimeson mixing and $C P$ violation occur at loop level and are therefore potentially subject to $\mathscr{O}(1)$ virtual corrections from New Physics.

The physics case of a Super $B$ factory with integrated luminosity of 50 to $75 \mathrm{ab}^{-1}$ has been discussed in detail in Refs. [4, 5]. In general, observables which are predicted by the Standard Model with small uncertainty or which are vanishingly small are most likely to provide evidence for New Physics at such a machine. Among them are lepton flavor violating $\tau$ decays, direct $C P$ asymmetries in $B \rightarrow X_{s, d} \gamma$, in $\tau$ decays and in some non-leptonic $D$ decays, $C P$ violation in neutral charm meson mixing, the dilepton invariant mass at which the forward-backward asymmetry of $B \rightarrow X_{S} \ell^{+} \ell^{-}$vanishes, and lepton universality violating $B$ and $\tau$ decays.

However, there is no single "golden mode" as in the BaBar/Belle era. It is important to note that a Super $B$ factory can access a wide range of observables and that virtually any model of New Physics in the TeV range will affect at least some of them. Even in the "worst case" scenario of minimal flavor violation where the New Physics doesn't contain any new sources of flavor and $C P$ violation, the flavor-violating couplings in the Standard Model are sufficient to make flavor processes sensitive to the presence of new particles. The experimental sensitivities to some of these observables are given in Table 1.

\section{Accelerator}

The Super $B$ factory in Japan ("SuperKEKB") is based on the existing KEKB machine, which is an asymmetric energy collider consisting of an $8 \mathrm{GeV}$ electron and a $3.5 \mathrm{GeV}$ positron ring 
Table 1: Expected sensitivities at a Super $B$ factory with $50-75 a b^{-1}$ compared to current sensitivities for selected physics quantities. From Ref. [5].

\begin{tabular}{ccc}
\hline \hline Observable & Super B sensitivity & Current sensitivity \\
\hline $\sin (2 \beta)\left(J / \psi K^{0}\right)$ & $0.005-0.012$ & 0.025 \\
$\gamma(D K)$ & $1-2^{\circ}$ & $\approx 31^{\circ}$ (CKMfitter) \\
$\alpha(\pi \pi, \rho \pi, \rho \rho)$ & $1-2^{\circ}$ & $\approx 15^{\circ}$ (CKMfitter) \\
$\left|V_{u b}\right|(\mathrm{excl})$ & $3-5 \%$ & $\approx 18 \%$ (PDG review) \\
$\left|V_{u b}\right|(\mathrm{incl})$ & $3-5 \%$ & $\approx 8 \%$ (PDG review) \\
\hline $\bar{\rho}$ & $1.7-3.4 \%$ & $-12 \%$ \\
$\bar{\eta}$ & $0.7-1.7 \%$ & $4.6 \%$ \\
\hline$S\left(\phi K^{0}\right)$ & $0.02-0.03$ & 0.17 \\
$S\left(\eta^{\prime} K^{0}\right)$ & $0.01-0.02$ & 0.07 \\
$S\left(K_{S} K_{S} K^{0}\right)$ & $0.02-0.03$ & 0.20 \\
$\mathscr{B}(B \rightarrow \tau \nu)$ & $3-4 \%$ & $30 \%$ \\
$\mathscr{B}(B \rightarrow \mu v)$ & $5-6 \%$ & not measured \\
$\mathscr{B}(B \rightarrow D \tau \nu)$ & $2-2.5 \%$ & $31 \%$ \\
\hline$A_{C P}(b \rightarrow s \gamma)$ & $0.004-0.005$ & 0.037 \\
$A_{C P}(b \rightarrow s \gamma+d \gamma)$ & 0.01 & 0.12 \\
$\mathscr{B}\left(B \rightarrow X_{d} \gamma\right)$ & $5-10 \%$ & $\approx 40 \%$ \\
$\mathscr{B}(B \rightarrow \rho \gamma) / \mathscr{B}\left(B \rightarrow K^{*} \gamma\right)$ & $3-4 \%$ & 0.24 \\
$S\left(K_{S} \pi^{0} \gamma\right)$ & $0.02-0.03$ & 0.67 \\
$S\left(\rho^{0} \gamma\right)$ & $0.08-0.12$ & $23 \%$ \\
$\mathscr{B}\left(B \rightarrow X_{S} \ell^{+} \ell^{-}\right)$ & $4-6 \%$ & not measured \\
$A^{F B}\left(B \rightarrow X_{S} \ell^{+} \ell^{-}\right)_{s 0}$ & $4-6 \%$ & not measured \\
$\mathscr{B}(B \rightarrow K v \bar{v})$ & $16-20 \%$ & $\approx 20^{\circ}$ \\
\hline$\phi_{D}$ & $1-2^{\circ}$ & not seen, $<5.0 \times 10^{-8}$ \\
$\mathscr{B}(\tau \rightarrow \mu \gamma)$ & $2-8 \times 10^{-9}$ & not seen, $<5.1 \times 10^{-8}$ \\
\hline $\mathscr{B}(\tau \rightarrow \mu \mu \mu)$ & $0.2-1 \times 10^{-9}$ & \\
$\mathscr{B}(\tau \rightarrow \mu \eta)$ & $0.4-4 \times 10^{-9}$ & \\
\hline \hline
\end{tabular}

located on the premises of the KEK lab in Tsukuba. KEKB is in operation since 1999 and has achieved a luminosity of $2.1 \times 10^{34} \mathrm{~cm}^{-2} \mathrm{~s}^{-1}$ in 2009 . For SuperKEKB this value needs to be increased by more than an order of magnitude (Fig. 1). The goals for the integrated luminosity are $10 \mathrm{ab}^{-1}$ for an initial operating phase and $50 \mathrm{ab}^{-1}$ by 2020 .

To achieve these numbers, two upgrade strategies have been proposed: the high current and the low emittance option. To understand these different concepts, let us consider the following expression for the luminosity,

$$
L=\frac{\gamma_{e \pm}}{2 e r_{e}}\left(1+\frac{\sigma_{y}^{*}}{\sigma_{x}^{*}}\right)\left(\frac{I_{e \pm} \xi_{y, e \pm}}{\beta_{y}^{*}}\right)\left(\frac{R_{L}}{R_{\xi_{y}}}\right) .
$$



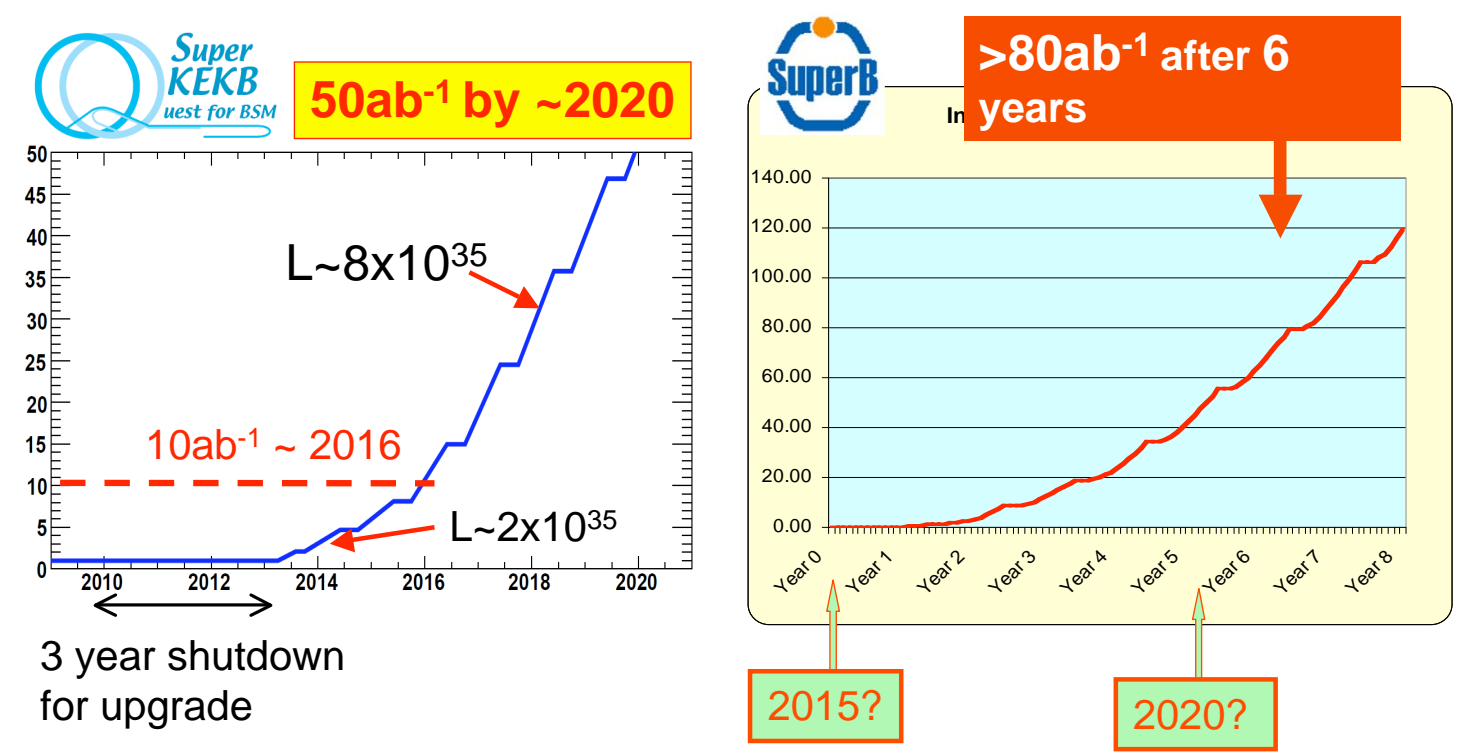

Figure 1: Luminosity prospects for SuperKEKB in Japan and SuperB in Italy.

Here, $\gamma_{e \pm}$ is a relativistic factor, $r_{e}$ is the classical electron radius, $\sigma_{x}^{*}$ and $\sigma_{y}^{*}$ are the horizontal and vertical beam sizes at the interaction point (IP), $I_{e \pm}$ are the stored currents, $\xi_{y, e \pm}$ is the beam-beam parameter, $\beta_{y}^{*}$ is the vertical $\beta$ function at the IP, and $R_{L} / R_{\xi_{y}}$ is a reduction factor reflecting the crossing angle.

The high current scheme has been intensely studied in the Letter of Intent of the KEK Super $B$ factory [6]. Here, high luminosities of about $5 \times 10^{35} \mathrm{~cm}^{-2} \mathrm{~s}^{-1}$ are achieved mainly by increasing the current in the electron (positron) ring from $1.1 \mathrm{~A}(1.6 \mathrm{~A})$ to $4.1 \mathrm{~A}(9.4 \mathrm{~A})$ which requires an upgrade of the RF system. In addition, the installation of the so-called crab cavities that tilt the bunches in the beams to obtain head-on collisions despite a finite crossing angle at IP, improve the beam-beam parameter $\xi_{y}$. Redesign of the interaction region leads to a reduction of the $\beta$ function at the IP (Table 2). To mitigate the problems that come with high beam currents (such as electron cloud instabilities and energy loss to higher order modes) new beam pipes and super-conducting HOM absorbing cavities will be installed. The drawbacks of this design are clearly the high RF wall plug power (and thus high operating costs) and the high levels of background in the experiment.

Therefore, a new scheme, the low emittance option, has been proposed, that foresees a drastic squeezing of the IP beam size $\sigma_{y}^{*}$ from $1.1 \mu \mathrm{m}$ at KEKB to $0.08 \mu \mathrm{m}$ at SuperKEKB. Here, a luminosity of $8 \times 10^{35} \mathrm{~cm}^{-2} \mathrm{~s}^{-1}$ can be achieved with considerably lower beam currents (Table 2). On the hardware side, this scheme requires a replacement of the long TRISTAN dipoles with shorter ones and a new interaction region with new final focusing quadrupoles near the IP. Also the injector complex needs to be modified to obtain low emittance electrons and positrons for injection. In November 2009, a solution for the machine optics was found, consistent with constraints from the existing tunnel and yielding reasonable beam lifetimes. The low emittance option is thus now the baseline for the SuperKEKB design.

The Italian Super $B$ factory ("SuperB") will be newly built either on the Tor Vergata site 
Table 2: Machine parameters for the present KEKB collider and for SuperKEKB (preliminary). Refer to the text for an explanation of the parameters.

\begin{tabular}{l|c|c|c|c}
\hline \hline Parameter & \multicolumn{2}{|c|}{ KEKB } & \multicolumn{2}{c}{ SuperKEKB } \\
LER/HER & design & achieved & high-current & low emittance \\
\hline$\beta_{y}^{*}(\mathrm{~mm})$ & $10 / 10$ & $5.9 / 5.9$ & $3 / 6$ & $0.24 / 0.37$ \\
$\sigma_{y}^{*}(\mu \mathrm{m})$ & 1.9 & 1.1 & $0.85 / 0.73$ & $0.084 / 0.072$ \\
$\xi_{y}$ & 0.052 & $0.101 / 0.096$ & $0.3 / 0.51$ & $0.09 / 0.09$ \\
$I_{\text {beam }}(\mathrm{A})$ & $2.6 / 1.1$ & $1.62 / 1.15$ & $9.4 / 4.1$ & $3.6 / 2.1$ \\
$L\left(10^{34} \mathrm{~cm}^{-2} \mathrm{~s}^{-1}\right)$ & 1 & 2.1 & 53 & 80 \\
\hline \hline
\end{tabular}

near Rome or at the INFN Laboratori Nazionali di Frascati. The machine concept relies on the "crabbed waist" collision scheme introduced by P. Raimondi [7] and foresees vertical beam sizes at the IP below $80 \mathrm{~nm}$. A distinctive feature is the use of a large Piwinski angle that allows to avoid the "hourglass" effect which would occur for $\beta_{y}^{*}$ much smaller than the bunch length $\sigma_{z}$. In the crabbed waist scheme the particles in the bunches collide in the minimum $\beta_{y}$ region and luminosities of $10^{36} \mathrm{~cm}^{-2} \mathrm{~s}^{-1}$ can be achieved (Fig. 1). This principle has recently been confirmed at the DA $\Phi N E$ collider in Frascati [8].

\section{Detector}

The detector at the Japanese Super $B$ factory ("Belle-II") will be built by upgrading the present Belle apparatus [1]. For the Italian SuperB detector, many components of the present BaBar spectrometer [2] will be reused. The aim of these upgrade programs (besides replacing aging components) is to cope with new requirements at a Super $B$ factory:

- Higher physics and background rate,

- Improved radiation tolerance, and

- Better detector hermeticity, e.g., for lepton flavor violation searches.

The baseline design of the Belle-II detector [6,9] foresees a replacement of the Belle 4-layer silicon vertex detector with a 2-layer DEPFET pixel device and a 4-layer silicon strip detector. DEPFET is a novel technology originally developed for vertex detection at the International Linear Collider (ILC) that has now reached a high level of maturity [10]. For Belle-II, the use of very thin $(50 \mu \mathrm{m})$ detectors with a pitch around $38 \times 50 \mu \mathrm{m}^{2}$ is planned. The strip layers consist of double-sided silicon strip sensors (DSSD) read out by fast APV25 chips, originally designed for the CMS experiment at the LHC. The Belle-II silicon detector will cover the radii between $1.3 \mathrm{~cm}$ and $14 \mathrm{~cm}$.

Also the Belle drift chamber will be replaced with a new one with smaller cell size (higher granularity) to cope with the higher occupancy. Particle identification will be provided by a Timeof-Propagation (TOP) counter in the barrel region and a proximity focusing Cherenkov ring imaging counter with aerogel radiators in the forward endcap (ARICH). The TOP measures the time 
that the internally reflected light travels down the quartz bar and one spatial coordinate along the bar.

The electromagnetic calorimeter has to cope with higher backgrounds. It is planned to replace the crystals in the endcap regions with pure CsI. For the entire device, new electronics with waveform sampling will be installed. The Belle muon system in the flux return of the magnet based on resistive plate chambers will probably remain unchanged in the barrel region. In the endcaps it will be replaced with scintillator strips.

Regarding the detector design for the Italian SuperB [7], the current BaBar silicon vertex tracker cannot operate at $L=10^{36} \mathrm{~cm}^{-2} \mathrm{~s}^{-1}$ and the drift chamber has reached the end of its design lifetime. The silicon device will thus be replaced, placing the inner-most layer at a radius of roughly $1.2 \mathrm{~cm}$. This innermost layer will be constructed of either silicon striplets or MAPS sensors. Likewise the cell size and geometry of the new drift chamber will be driven by occupancy considerations. The quartz bars of the DIRC particle identification device will be reused but the readout will be replaced with either faster PMTs in the current water tank or multichannel plate (MCP) photon detectors in a focusing configuration. A forward particle identification system using a time-of-flight might be added. The BaBar barrel calorimeter will be reused but complemented by a new forward calorimeter using LYSO (lutetium yttrium orthosilicate) crystals for better radiation hardness and reduced sensitivity to beam backgrounds. The flux-return will be augmented with additional absorber to increase the number of interaction lengths for muons to roughly 7 . The baseline design for the instrumentation of the flux-return are scintillator bars similar to the active detector of the MINOS experiment.

\section{Summary}

We have reviewed the status of the two Super $B$ factory projects, SuperKEKB/Belle-II in Japan and SuperB in Italy, both aiming at investigating heavy flavor physics and the Cabibbo-KobayashiMaskawa mechanism with data sets larger than $50 \mathrm{ab}^{-1}$. Both projects have made significant progress in the last years.

For SuperKEKB/Belle-II, the most important steps were: the Letter of Intent for the KEK Super $B$ factory in June 2004 [6]; the project becomes part of the KEK road-map in January 2008; the Belle-II collaboration is officially formed in December 2008; SuperKEKB/Belle-II receive an R\&D budget in May 2009; and the project receives "preliminary approval" by the new Japanese government in January 2010 and starts the construction phase. The detailed Technical Design Report (TDR) is expected for the first half of 2010.

SuperB submitted a Conceptual Design Report (CDR) [7] in September 2007, signed by 320 individuals for 85 institutions. A machine advisory committee was formed in June 2008. In December 2008, the TDR phase was approved and funded by INFN. The detailed technical design document is expected for the end of 2010.

\section{References}

[1] A. Abashian et al. [Belle Collaboration], Nucl. Instrum. Meth. A 479, 117 (2002).

[2] B. Aubert et al. [BABAR Collaboration], Nucl. Instrum. Meth. A 479, 1 (2002). 
[3] M. Kobayashi and T. Maskawa, Prog. Theor. Phys. 49, 652 (1973).

[4] T. Browder, M. Ciuchini, T. Gershon, M. Hazumi, T. Hurth, Y. Okada and A. Stocchi, JHEP 0802, 110 (2008) [arXiv:0710.3799 [hep-ph]].

[5] T. E. Browder, T. Gershon, D. Pirjol, A. Soni and J. Zupan, arXiv:0802.3201 [hep-ph].

[6] S. Hashimoto et al., KEK-REPORT-2004-4.

[7] M. Bona et al., arXiv:0709.0451 [hep-ex].

[8] D. Alesini et al., SB-NOTE-GEN-2008-00.

[9] I. Adachi et al. [sBelle Design Group], arXiv:0810.4084 [hep-ex].

[10] J. J. Velthuis et al., Nucl. Instrum. Meth. A 579, 685 (2007). 